Как дефицит железа (Fe), так и гипергликемия широко распространены во всем мире среди беременных женщин. По последним данным Американской ассоциации диабета (ADA), распространенность гестационного сахарного диабета (ГСД) возросла и достигает 15-20\%. В настоящее время появляется все больше доказательств того, что существует связь между метаболизмом Fе и гомеостазом глюкозы. Изучение изменений показателей обмена Fе в сыворотке беременных с нарушением толерантности к глюкозе и ГСД играет важную роль в расширении понимания патогенеза данных состояний. Гипотеза о том, что избыток Fе повышает риск развития ГСД, побудила нас выполнить обзор и оценить потенциальную связь между повышенным уровнем Fе и риском развития ГСД. Цель - обобщить имеющиеся данные о корреляции статуса Fе в организме с ГСД. Становится все более общепризнанным, что избыточное накопление Fе связано с повышенным риском диабета. Данные о взаимосвязи между сывороточным ферритином и ГСД в российской популяции отсутствуют, тогда как раннее выявление риска развития ГСД будет иметь большое значение для предотвращения заболевания и связанных с ним последствий для здоровья. Роль статуса Fе в качестве биомаркера ГСД в популяциях высокого риска представляет собой интерес как для прогностических и диагностических мероприятий, так и для терапевтических вмешательств. Для лучшего понимания того, увеличивает ли избыток Fе риск развития ГСД, необходимы исследования, которые раскроют роль Fе в механизмах развития ГСД.

КЛЮЧЕВЫЕ СЛОВА: гестачионный сахарный диабет; железо; ферритин; гемоглобин; гепсидин; факторы риска

\title{
THE ROLE OF IRON METABOLISM INDICATORS IN PREDICTING GESTATIONAL DIABETES MELLITUS
}

\author{
๑) Ayarpi O. Torosyan*, Ekaterina V. Loginova, Chelebi G. Gagaev
}

Peoples' Friendship University of Russia, Moscow, Russia

Iron (Fe) deficiency and hyperglycaemia are both widely found throughout the world among pregnant women. According to the latest data from the American Diabetes Association (ADA), the prevalence of gestational diabetes mellitus (GDM) has increased and reaches $15-20 \%$. Nowadays, there is growing evidence that a link between Fe metabolism and glucose homeostasis exists. The study of changes in the indicators of Fe metabolism in the serum of pregnant women with glucose intolerance and GDM plays an important role in expanding the understanding of the pathogenesis of these conditions. The hypothesis that excess Fe increases the risk of developing GDM has prompted us to review and evaluate the potential relationship between elevated Fe levels and the risk of developing GDM. The aim is to integrate all available data on the correlation between GDM and Fe status in the body. It is increasingly being recognised that excess Fe accumulation in the body is associated with an increased risk of diabetes. There is no available data on the relationship between serum ferritin and GDM in the Russian population, while early identification of the risk of GDM development will be of great importance for its related health effects and prevention. The role of Fe status as a GDM biomarker in high-risk populations is of interest, both for prognostic and diagnostic measures, and for therapeutic interventions. For a better understanding of whether an excess of Fe increases the risk of developing GDM, studies are needed to reveal the role of Fe in the mechanisms of GDM development.

KEYWORDS: gestational diabetes; iron; ferritin; haemoglobin; hepcidin; risk factors

Гестационный сахарный диабет (ГСД) является наиболее распространенным нарушением обмена веществ во время беременности и влияет на перинатальные исходы и отдаленные результаты как у новорожденных, так и у их матерей $[1,2]$. Согласно последним данным Американской диабетической ассоциации (ADA), его распространенность возросла и достигает 15-20\% [3].

Этиология ГСД является многофакторной и до сих пор окончательно не установлена [4]. Как профилактика, так и своевременная диагностика и лечение ГСД возможны путем выявления факторов риска (ФР), в частности, тех, которые влияют на метаболизм глюкозы [5]. Изучение изменений содержания химических элементов в сыворотке беременных с нарушением толерантности к глюкозе и ГСД играет важную роль в расширении понимания патогенеза данных состояний [6].

Как и гипергликемия, дефицит железа (Fe) широко распространен во всем мире среди беременных женщин $[7,2]$. Fе является одним из микроэлементов, которые необходимы для человека, и потребность в Fe во время беременности увеличивается [8]. Выявленная связь между избытком Fe и сахарным диабетом 2 типа наталкивает на мысль, что повышение уровня Fe также играет роль в развитии ГСД [9]. 
ФР, связанные с ГСД, еще не полностью идентифицированы [10]. Поскольку за последние 20 лет заболеваемость ГСД увеличивалась, при этом примерно в половине случаев зарегистрирован также избыточный вес женщин, перспективен поиск других потенциально контролируемых ФР [11].

Большинство руководств и рекомендаций по диагностике ГСД предполагают скрининг на 24-28-й неделе беременности, ссылаясь на недостаток современных данных для того, чтобы рекомендовать проведение перорального глюкозотолерантного теста (ПГТТ) на более ранних сроках [12]. ПГТТ отнимает много времени и обременителен как для пациентов, так и для медицинского персонала. Кроме этого, проведение скринингового теста в конце II триместра при положительном результате оставляет мало времени для эффективной коррекции $[12,13]$.

Многие исследователи делают акцент на недостаточной чувствительности ПГТТ для обнаружения ГСД [2]. Поэтому предлагаются другие, потенциально более перспективные тесты (фетальный инсулин и др.) для выявления ГСД и прогнозирования неблагоприятного исхода беременности. Однако широко проводить инсулинометрию околоплодных вод у беременных женщин неприемлемо. В связи с этим актуален поиск других простых анализов крови, которые помогут неинвазивно идентифицировать женщин с высоким риском ГСД $[12,13]$.

\section{ПОКАЗАТЕЛИ ОБМЕНА ЖЕЛЕЗА КАК МАРКЕРЫ ПРОГНОЗИРОВАНИЯ ГЕСТАЦИОННОГО САХАРНОГО ДИАБЕТА}

Обсуждается вопрос о том, связано ли избыточное содержание эндогенного и экзогенного Fe с риском манифестации ГСД. Но до сих пор исследования были неоднородны в отношении выбора показателей для оценки уровня Fе в организме, критериев диагностики ГСД, дизайна исследования и демографических характеристик исследуемых [14]. В отличие от данных о количестве поступающего в организм Fе, его уровень в крови более достоверно отражает обмен Fе и может дать важную информацию о его роли в патогенезе ГСД [15].

Различные биомаркеры - гемоглобин (Hb), сывороточное $\mathrm{Fe}$, ферритин, гепсидин - использовались для изучения взаимосвязи между состоянием Fe в организме и ГСД [16]. Исследования по этому вопросу довольно многочисленны, но противоречивы в своих выводах.

Впервые о корреляции между уровнем ферритина в сыворотке и нарушением углеводного обмена среди беременных женщин было упомянуто в работе T. Lao и K. Tam в 1997 г. [17]. Позже они же, предположив, что у женщин с железодефицитной анемией вероятность развития ГСД ниже, провели ретроспективное исследование и продемонстрировали, что железодефицитная анемия сопровождается снижением риска ГСД [18]. Напротив, значение Нb более 130 г/л при постановке на учет является независимым ФР развития ГСД [19].

S. Behboudi-Gandevani и соавт. на основе продольного проспективного исследования доказали, что содержание Fe в материнской сыворотке выше 100 мкг/дл на 14-20-й неделе беременности является оптимальным пороговым значением для прогнозирования ГСД с чувствительностью и специфичностью 80,6 и 50,7\% соответственно [20].
Сывороточный ферритин широко используется в качестве показателя запасов Fе в организме [21, 22]. Однако, по результатам другого исследования, различий в уровне Fe и общей железосвязывающей способности сыворотки между женщинами с ГСД и контрольной группой обнаружено не было, хотя высокий уровень ферритина в сыворотке увеличивал риск ГСД в 2,4 раза [1].

О положительной корреляции между повышенным уровнем ферритина и показателями ПГТТ сообщали и другие исследователи [1, 8, 17, 23-26, 27]. В частности, Y. Cheng и соавт. (2020 г.) отмечают положительную линейную связь между уровнями сывороточного ферритина и гликемии через 1 ч после нагрузки глюкозой при проведении ПГТТ [8].

Учеными рассмотрена также взаимосвязь между уровнем Fе и риском развития ГСД в зависимости от триместра беременности. Rawal и соавт. исследовали уровень Fe у матери в I и II триместрах беременности с последующей оценкой риска развития ГСД. Ими отмечена значимая положительная корреляция между уровнем ферритина в 10-14 нед и манифестацией ГСД в последующем [28]. Результаты проведенного метаанализа также показали, что связь между уровнями $\mathrm{Hb}$, ферритина и риском развития ГСД значительна, когда эти показатели определяются в I триместре беременности [12].

Таким образом, определение концентрации $\mathrm{Hb}$ и/или ферритина в ранние сроки беременности может составить основу эффективного раннего скрининга по развитию ГСД $[12,15]$.

Взаимосвязь биомаркеров запаса Fe в организме, определенных в I триместре беременности, с риском развития ГСД была оценена в датской популяции. На сроке беременности 9,4ะ3,2 нед определяли уровень ферритина и растворимого рецептора трансферрина (sTfR) с учетом системного воспаления и окислительного стресса (ОС), измеряя С-реактивный белок (СРБ), окисленный липопротеинами низкой плотности. Концентрация как ферритина, так и sTfR на ранних сроках беременности была значительно выше в группе с ГСД, чем в контрольной. Положительная связь концентрации STfR с риском развития ГСД была ослаблена и стала незначительной после учета индекса массы тела (ИМТ) до беременности. Однако ферритин был положительно и значимо связан с риском развития ГСД даже после корректировки результатов в соответствии с основными ФР ГСД, включая ИМТ до беременности [21].

В исследовании «случай-контроль», помимо уровней $\mathrm{Hb}$, ферритина и Fe, в основной группе с ГСД также были значительно повышены такие показатели, как средний объем эритроцитов, среднее содержание Нb в эритроците и насыщение трансферрином [23].

Однако, по данным нескольких других работ, между группами с ГСД и без него по средним значениям $\mathrm{Hb}$, гематокрита, Fе, ферритина, общей железосвязывающей способности сыворотки и трансферрина различий найдено не было [6, 29, 30]. Более того, в исследовании F. Akhlaghi и соавт. в группе женщин с ГСД концентрация Fe в сыворотке крови была значительно ниже, чем в контрольной группе [31].

Wang и соавт. [2018] также рассмотрели влияние уровня Fе на макросомию и выявили, что высокий уровень ферритина может быть независимым ФР развития 
макросомии [2, 24]. Частота возникновения макросомии была выше у беременных с уровнем ферритина плазмы >70 нг/л [2].

Тем не менее, некоторое недоверие к концентрации ферритина в качестве показателя запасов Fе в организме связано с тем, что его уровень в организме также увеличивается при системном воспалении [21]. Все больше доказательств в пользу того, что ГСД обусловлен воспалительным процессом, что также может объяснить взаимосвязь между уровнем ферритина и риском развития ГСД [4]. Повышенные уровни ферритина в сыворотке женщин с ГСД, возможно, являются результатом иммунного ответа воспалительных цитокинов, а не высоким показателем запасов Fe [32]. Так, V. Soubasi и соавт., выявив более высокий уровень ферритина при родах у женщин с ГСД, связали этот факт не столько с повышенным уровнем Fe в организме, сколько с системным воспалением [25].

Концентрация STfR также является индикатором состояния $\mathrm{Fe}$, поскольку дефицит Fе приводит к избыточной экспрессии концентраций STfR, тогда как STfR снижается в присутствии перегрузки железом [21].

B работе Yang и соавт. уровни STfR в материнской сыворотке были значительно повышены в группе ГСД по сравнению с группой без ГСД. Уровни ферритина в пуповинной крови в основной группе также были существенно выше. Однако уровни материнского $\mathrm{Hb}$ и ферритина, а также STfR пуповины не различались между группами [32]. По результатам исследования «случай-контроль» величина соотношения sTfR/ферритин была обратно пропорциональна риску ГСД [28].

Отражает ли sTfR связь между более высокими концентрациями Fe и ГСД, в настоящее время неясно, и это требует дальнейшего изучения [21].

Результаты нескольких исследований подтверждают идею о том, что гепсидин (пептид из 25 аминокислот) является основным регулятором гомеостаза Fе, контролируя его всасывание из пищевых источников в кишечнике, рециркуляцию из макрофагов и накопление в печени $[15,33,34]$. Исследования роли гепсидина - гормона, регулирующего метаболизм $\mathrm{Fe}$, в патогенезе нарушений углеводного обмена продолжаются [35].

Согласно данным Derbent и соавт. [2013], уровень гепсидина в сыворотке крови был значительно повышен у беременных с ГСД. Корреляции между гепсидином и другими показателями метаболизма $\mathrm{Fe}$ (Hb, сывороточное Fe и ферритин) обнаружено не было, однако выявлена положительная взаимосвязь между уровнем гепсидина и параметрами метаболизма глюкозы (уровни глюкозы и инсулина в крови натощак и результаты ПГТТ) [33].

Согласно данным литературы, нарушение синтеза гепсидина у пациентов с диабетом проявлялось как снижением его продукции и синдромом перегрузки $\mathrm{Fe}$, так и повышением синтеза и развитием анемии хронических заболеваний [35]. Хотя гепсидин привлекает внимание как главный регулятор гомеостаза $\mathrm{Fe}$ вопрос о том, какие показатели для оценки статуса Fe являются оптимальными, остается предметом дискуссий [15].

Наиболее распространенным состоянием первичной перегрузки $\mathrm{Fe}$ является наследственный гемохроматоз, связанный с геном гемохроматоза (HFE). Повышенное усвоение $\mathrm{Fe}$, обусловленное неспособностью регулировать кишечную абсорбцию, приводит к отложению $\mathrm{Fe}$ в различных органах, включая поджелудочную железу. Распространенность двух наиболее распространенных мутаций, C282Y и H63D, была определена у пациентов с ГСД и сравнивалась с контрольной группой. У пациентов основной группы частота аллельных мутаций C282Y (7,7\%) была выше, чем у беременных без ГСД (2,9\%; $\mathrm{p}=0,04)$, в то время как частота мутаций H63D не отличалась. Таким образом, эти данные свидетельствуют о том, что мутации в гене HFE способствуют развитию ГСД, что позволяет предположить генетическую предрасположенность к развитию ГСД. В связи с этим носители мутации С282Y должны быть тщательно обследованы на протяжении всей беременности для своевременного выявления и лечения ГСД [36].

Генетическая предрасположенность также подтверждается обнаружением более высоких запасов $\mathrm{Fe}$ и частоты возникновения ГСД у страдающих гетерозиготной формой гемоглобинопатий (талассемия, серповидноклеточная анемия), чем в контрольной группе [37].

Роль Fe важна для ключевых процессов в поддержании метаболического гомеостаза, включая функционирование и дифференцировку адипоцитов, а также передачу сигналов инсулина в печени и мышцах. Так, имеющиеся данные свидетельствуют о том, что избыток Fe способствует дисфункции адипоцитов и нарушению передачи сигналов инсулина в мышцах и печени [38].

Экспериментальные исследования на мышах продемонстрировали влияние избытка Fe на продукцию адипокинов: повышающее в отношении резистина и понижающее в отношении адипонектина, также способствующее прогрессированию инсулинорезистентности [39].

Fe может играть роль в непереносимости глюкозы через два потенциальных механизма: воспаление и избыток Fе. Известно, что воспаление повышает уровень ферритина и является патофизиологическим процессом, лежащим в основе хронической анемии. Состояния избытка Fе в организме, такие как гемохроматоз, вызывают диабет через отложение Fe в поджелудочной железе [13].

Одной из теорий, объясняющих роль Fе в нарушении углеводного обмена, является ферротоксичность. Будучи сильным прооксидантом, свободное Fе катализирует реакции, продуктами которых являются активные формы кислорода, в том числе гидроксильные радикалы, повышая тем самым уровень ОС [2, 7, 21, 28, 40].

Реакция Фентона: $\mathrm{Fe}^{2+}+\mathrm{H}_{2} \mathrm{O}_{2} \rightarrow \mathrm{Fe}^{3+}+\mathrm{OH}^{-}+\cdot \mathrm{OH}$.

ОС, в свою очередь, вызывает повреждение и апоптоз $\beta$-клеток и, следовательно, способствует нарушению синтеза и секреции инсулина [28]. Учитывая низкую антиоксидантную защиту, $\beta$-клетка поджелудочной железы особенно уязвима и восприимчива к окислительному повреждению, что в итоге приводит к нарушению синтеза инсулина $[12,14,21,35,38,40,41]$. В $\beta$-клетках человека циркадные часы контролируют секрецию инсулина. Предполагается, что избыток Fe нарушает циркадные часы в $\beta$-клетках [38].

Вполне вероятно также, что избыток Fе, влияя на чувствительность к инсулину во всех тканях, способствует развитию диабета путем нарушения метаболизма глюкозы и возникновения инсулинорезистентности [38]. 
Животные модели показали, что перегрузка Fe приводит к инсулинорезистентности и выработке глюкозы в печени [14]. Влияя на синтез и секрецию инсулина и усиливая окисление липидов, избыток Fе снижает использование глюкозы в мышцах и увеличивает глюконеогенез в печени, что приводит к опосредованной печенью резистентности к инсулину [23]. Накопление Fе нарушает инсулин-индуцированный транспорт глюкозы в мышцы и адипоциты [28]. Кроме того, избыточное содержание Fe в крови усугубляет инсулинорезистентность за счет подавления образования адипонектина и усиления продукции резистина [35].

Известно, что в течение нескольких лет после беременности, осложненной ГСД, женщины имеют риск развития сахарного диабета 2 типа из-за прогрессирования дисфункции $\beta$-клеток, которая усугубляется инсулинорезистентностью. Канадские ученые проанализировали взаимосвязь между ферритином и гомеостазом глюкозы в первые годы после родов у женщин с ГСД и без. Согласно полученным результатам, ни исходный уровень ферритина, ни его изменение через 1 и 3 года не были связаны с какими-либо из следующих показателей через 3 года после родов: индексы инсулинорезистентности (HOMA-IR), инсулиночувствительности (Matsuda), чувствительности к секреции инсулина-2 (ISSI-2), инсулиногенный индекс (IGI)/HOMA-IR, уровень глюкозы натощак, результаты ПГТТ. Авторы пришли к заключению, что ферритин, по-видимому, не играет существенной роли в определении риска диабета в первые годы после беременности, осложненной ГСД [42].

\section{ПРОФИЛАКТИКА И ЛЕЧЕНИЕ ЖЕЛЕЗОДЕФИЦИТНОЙ АНЕМИИ И ГЕСТАЦИОННОГО САХАРНОГО ДИАБЕТА}

Дефицит Fe широко распространен среди беременных и остается глобальной проблемой здравоохранения [14]. Анемия обычно рассматривается как ФР неблагоприятных исходов, приводящий к осложнениям как со стороны матери, так и со стороны плода $[41,43]$. Однако была выдвинута гипотеза, что железодефицитная анемия (ЖДА) снижает частоту ГСД. Так, по результатам метаанализа шести исследований с участием 15157 участников, у беременных с ЖДА вероятность развития ГСД на 39\% ниже [41].

Согласно рекомендациям ВО3 по ведению беременности, для профилактики анемии, послеродового сепсиса, рождения маловесных детей и преждевременных родов беременным необходимо ежедневное потребление 30-60 мг Fe [43].

Обнаружив значительную связь между потреблением гемового Fe и развитием ГСД ученые предположили, что беременным следует избегать употребления чрезмерного количества пищи, богатой гемовым Fe, особенно тем, у кого есть другие уже известные ФР ГСД, такие как ожирение, семейный анамнез диабета и пожилой возраст [22].

В развитых странах профилактический прием препаратов Fе назначается беременным в зависимости от их уровня сывороточного ферритина. Более того, беременным с высоким уровнем ферритина поливитамины рекомендуются с особой осторожностью [2].
Последствия рутинного введения железосодержащих добавок всем беременным требуют более тщательного изучения, поскольку обнаружена значительная связь между диабетом и повышенным уровнем ферритина в сыворотке [1].

Zhu и соавт. [2018] выявили положительную связь между приемом железосодержащих добавок до беременности и риском развития ГСД. В данной группе женщин риск развития ГСД был на 57\% выше по сравнению с теми, кто не получал препараты Fе. Однако употребление железосодержащих добавок в I и II триместрах никак не повлияло на развитие ГСД [44].

Противоположные результаты получены в Финляндии. Было опубликовано крупномасштабное рандомизированное контролируемое исследование влияния приема добавок Fе на ГСД. Результаты продемонстрировали, что рутинная профилактическая добавка Fe (100 мг ежедневно в течение всей беременности независимо от наличия анемии) не увеличивала риск развития ГСД [11]. Данные метаанализа и еще одного ретроспективного исследования показали, что прием препаратов Fе не увеличивает риск развития ГСД $[22,45]$.

Таким образом, остается неясным, может ли окисление липидов и повреждение ДНК из-за приема препаратов и избытка Fе в организме способствовать развитию ГСД $[15,28]$. Профилактические дозы Fe ( $\leq 60$ мг в день) во время беременности для лиц с нормальным уровнем сывороточного Fе требуют дальнейших исследований [16].

В целом появляющиеся на сегодняшний день ограниченные данные свидетельствуют о том, что пищевое гемовое Fе положительно и в значительной степени связано с риском развития ГСД, тогда как результаты, касающиеся негемового $\mathrm{Fe}$, в целом неубедительны [15].

Пероральное назначение препаратов Fe беременным без анемии, вероятно, не оправдано для женщины с нормальным Нb в I триместре.

Принимая во внимание вышеописанное, можно заключить, что вопрос о назначении препаратов Fе должен решаться индивидуально, основываясь на уровне ферритина в плазме крови.

В так называемых странах 3-го мира общее улучшение питания и коррекция анемии могут быть факторами, способствующими увеличению распространенности ГСД. Однако это не следует истолковывать в пользу отказа от приема препаратов Fе для коррекции материнской анемии, важность которой бесспорна [18]. Данные о взаимосвязи между сывороточным ферритином и ГСД в российской популяции отсутствуют.

\section{ЗАКЛЮЧЕНИЕ}

Раннее выявление риска развития ГСД будет иметь большое значение для предотвращения заболевания и связанных с ним последствий для здоровья [12]. Потенциальная роль статуса Fe в качестве биомаркера ГСД в популяциях высокого риска представляет собой интерес как для прогностических и диагностических мероприятий, так и для терапевтических вмешательств [5]. Для лучшего понимания того, увеличивает ли избыток Fe риск развития ГСД, необходимы дальнейшие исследования, которые раскроют роль Fе в механизмах развития ГСД [9]. 


\section{ДОПОЛНИТЕЛЬНАЯ ИНФОРМАЦИЯ}

Конфликт интересов. Авторы декларируют отсутствие явных и потенциальных конфликтов интересов, связанных с публикацией настоящей статьи.
Участие авторов. Торосян А.О. - анализ литературы, написание и исправление рукописи, ответственность за целостность всех ее частей; Логинова Е.В. - поиск и анализ литературы, сбор и интерпретация данных; Гагаев Ч.Г. - доработка, внесение принципиальных изменений, окончательное утверждение версии рукописи.

\section{СПИСОК ЛИТЕРАТУРЫ | REFERENCES}

1. Amiri FN, Basirat Z, Omidvar S, et al. Comparison of the serum iron, ferritin levels and total iron-binding capacity between pregnant women with and without gestational diabetes. J Nat Sc Biol Med. 2013:4:302-305. doi: https://doi.org/10.4103/0976-9668.116977

2. Wang Z, Fan $\mathrm{H}$, Yang W, et al. Correlation between plasma ferritin level and gestational diabetes mellitus and its impact on fetal macrosomia. J Diabetes Investig. 2018;9:1354-1359. doi: https://doi.org/10.1111/jdi.12836

3. Standards of medical care in diabetes-2017. American Diabetes Association. Diabetes Care. 2017:40:S11-S24

4. Fu S, Li F, Zhou J, Liu Z. The relationship between body iron status, iron intake and gestational diabetes: A systematic review and meta-analysis. Medicine. 2016;95(2):e2383. doi: https://doi.org/10.1097/MD.0000000000002383

5. Zein S, Rachidi S, Hininger-Favier I. Is oxidative stress induced by iron status associated with gestational diabetes mellitus? J. Trace Elem. Med. Biol. 2014;28:65-69. doi: https://doi.org/10.1016/j.jtemb.2013.09.009

6. Wang $Y$, Tan M, Huang Z, et al. Elemental contents in serum of pregnant women with gestational diabetes mellitus. Biol. Trace Elem. Res. 2002;88:113-118. doi: https://doi.org/10.1385/BTER:88:2:113

7. Zein S, Rachidi S, Awada S, et al. High iron level in early pregnancy increased glucose intolerance. JTEMB. 2015;30:220-225.

doi: https://doi.org/10.1016/j.jtemb.2014.09.004

8. Cheng Y, LiT, He M, et al. The association of elevated serum ferritin concentration in early pregnancy with gestational diabetes mellitus: a prospective observational study. Eur J Clin Nutr. 2020;74(5):741-748. doi: https://doi.org/10.1038/s41430-019-0542-6

9. Khambalia AZ, Aimone A, Nagubandi P, et al. High maternal iron status, dietary iron intake and iron supplement use in pregnancy and risk of gestational diabetes mellitus: a prospective study and systematic review. Diabet Med. 2016;33(9):1211-21. doi: https://doi.org/10.1111/dme.13056

10. Amiri FN, Basirat Z, Omidvar S, et al. Comparison of the serum iron, ferritin levels and total iron-binding capacity between pregnant women with and without gestational diabetes. J Nat Sci Biol Med. 2013;4:302-305. doi: https://doi.org/10.4103/0976-9668.116977

11. Kinnunen $\mathrm{TI}$, Luoto R, Helin A, Hemminki E. Supplemental iron intake and the risk of glucose intolerance in pregnancy: Re-analysis of a randomised controlled trial in Finland. Matern Child Nutr. 2016;12(1):74-84. doi: https://doi.org/10.1111/mcn.12139

12. Fernández-Cao JC, Aranda N, Ribot B, et al. Elevated iron status and risk of gestational diabetes mellitus: A systematic review and meta-analysis. Matern Child Nutr. 2017;13(4):e12400. doi: https://doi.org/10.1111/mcn.12400

13. Powe CE. Early Pregnancy Biochemical Predictors of Gestational Diabetes Mellitus. Curr Diab Rep. 2017; 17(2):12. doi: https://doi.org/10.1007/s11892-017-0834-y

14. Kataria Y, Wu Y, Horskjær PH, et al. Iron Status and Gestational Diabetes - A Meta-Analysis. Nutrients. 2018;(10):621. doi: https://doi.org/10.3390/nu10050621

15. Zhang C, Rawal S. Dietary iron intake, iron status, and gestational diabetes. Am J Clin Nutr. 2017;106(6):1672S-1680S. doi: https://doi.org/10.3945/ajcn.117.156034

16. Zhuang T, Han H, Yang Z. Iron, Oxidative Stress and Gestational Diabetes. Nutrients. 2014;6:3968-3980. doi: https://doi.org/10.3390/nu6093968

17. Lao TT, Tam KF. Maternal serum ferritin and gestational impaired glucose tolerance. Diabetes Care. 1997;20(9):1368-1369. doi: https://doi.org/10.2337/diacare.20.9.1368

18. Lao TT, Ho LF. Impact of iron deficiency anemia on prevalence of gestational diabetes mellitus. Diabetes Care. 2004;27(3):650-656. doi: https://doi.org/10.2337/diacare.27.3.650

19. Lao TT, Chan LY, Tam KF, Ho LF. Maternal hemoglobin and risk of gestational diabetes mellitus in chinese women. Obstet Gynecol. 2002;99:807-812. doi: https://doi.org/10.1016/s0029-7844(02)01941-5
20. Behboudi-Gandevani S, Safary K, Moghaddam-Banaem L, et al. The relationship between maternal serum iron and zinc levels and their nutritional intakes in early pregnancy with gestational diabetes. Biol Trace Elem Res. 2013;154:7-13. doi: https://doi.org/10.1007/s12011-013-9703-y

21. Bowers KA, Olsen SF, Bao W, et al. Plasma concentrations of ferritin in early pregnancy are associated with risk of gestational diabetes mellitus in women in the Danish national birth cohort. J Nutr. 2016;146:1756-1761. doi: https://doi.org/10.3945/jn.115.227793

22. Zhao L, Lian J, Tian J, et al. Dietary intake of heme iron and body iron status are associated with the risk of gestational diabetes mellitus: a systematic review and meta-analysis. Asia Pac J Clin Nutr. 2017; 26(6):1092-1106. doi: https://doi.org/10.6133/apjen.022017.09

23. Afkhami-Ardekani M, Rashidi M. Iron status in women with and without gestational diabetes mellitus. J Diabetes Complications. 2009;23:194-198. doi: https://doi.org/10.1016/j.jdiacomp.2007.11.006

24. Chen X, Scholl TO, Stein TP. Association of elevated serum ferritin levels and the risk of gestational diabetes mellitus in pregnant women: The camden study. Diabetes Care. 2006;29:1077-1082. doi: https://doi.org/10.2337/dc06-0164

25. Soubasi V, Petridou S, Sarafidis K, et al. Association of increased maternal ferritin levels with gestational diabetes and intrauterine growth retardation. Diabetes \& Metabolism. 2010;36:58-63. doi: https://doi.org/10.1016/j.diabet.2009.06.010

26. Lao TT, Chan PL, Tam KF. Gestational diabetes mellitus in the last trimester - a feature of maternal iron excess? Diabetic Med. 2001;18:218-223. doi: https://doi.org/10.1046/j.1464-5491.2001.00453.x

27. Yadav A, Saini V, Kataria M, Jain A. Need of iron supplementation in gestational diabetes mellitus. Acta Endocrinologica. 2017;XIII(1):126-128. doi: https://doi.org/10.4183/aeb.2017.126

28. Rawal S, Hinkle SN, Bao W, et al. A longitudinal study of body iron status during pregnancy and risk of gestational diabetes: findings from a prospective, multiracial cohort. Diabetologia. 2017;60(2):249-257. doi: https://doi.org/10.1007/s00125-016-4149-3

29. Al-Saleh E, Nandakumaran M, Al-Shammari M, Al-Harouny A Maternal-fetal status of copper, iron, molybdenum, selenium, and zinc in patients with gestational diabetes. J Matern Fetal Neonatal Med. 2004;16:15-21. doi: https://doi.org/10.1080/14767050412331283139

30. Yeniel AO, Ergenoglu AM, Sanhal CY, et al. Does high maternal first trimester iron status have an effect on the $50 \mathrm{~g}$ oral glucose test? J Obstet Gynaecol. 2012;32:332-334. doi: https://doi.org/10.3109/01443615.2012.658894

31. Akhlaghi F, Bagheri SM, Rajabi O. A comparative study of relationship between micronutrients and gestational diabetes. ISRN Obstet Gynecol. 2012;2012(470419). doi: https://doi.org/10.5402/2012/470419 32.

32. Yang A, Zhao J, Lu M, et al. Expression of Hepcidin and Ferroportin in the Placenta, and Ferritin and Transferrin Receptor 1 Levels in Maternal and Umbilical Cord Blood in Pregnant Women with and without Gestational Diabetes. Int J Environ Res Public Health. 2016;13(766). doi: https://doi.org/10.3390/ijerph13080766

33. Derbent AU, Simavli SA, Kaygusuz I, et al. Serum hepcidin is associated with parameters of glucose metabolism in women with gestational diabetes mellitus. J. Matern. Fetal Neonatal Med. 2013;26:1112-1115. doi: https://doi.org/10.3109/14767058.2013.770462

34. Маянский Н.А., Семикина Е.Л. Гепцидин: основной регулятор обмена железа и новый диагностический маркер // Bопросы диагностики в педиатрии. - 2009. - №1. - C. 18-23. [Majanskij NA, Semikina EL. Gepcidin: osnovnoj reguljator obmena zheleza i novyj diagnosticheskij marker. Voprosy diagnostiki v pediatrii. 2009;1:18-23. (In Russ.)]. 
35. Саприна Т.В., Зима А.П., Мусина Н.Н., и др. Патогенетические аспекты нарушения метаболизма гепсидина и феррокинетики при патологии углеводного обмена // Сахарный диабет. 2018. - T. 21. — №6. - C. 506-512. [Saprina TV, Zima AP, Musina NN, et al. Pathogenetic aspects of hepcidin metabolism and ferrocinetics dysregulation in carbohydrate metabolism disorders. Diabetes Mellitus. 2018;21(6):506-512 (In Russ.)]. doi: https://doi.org/10.14341/DM9378

36. Cauza E, Hanusch-Enserer U, Bischof M, et al. Increased c282y heterozygosity in gestational diabetes. Fetal Diagn Ther. 2005;20:349-354. doi: https://doi.org/10.1159/000086811

37. Bencaiova G, Krafft A, Burkhardt T, Zimmermann R. Hemoglobinopathies, body iron stores and gestational diabetes mellitus. Haematologica. 2005;90:1138-1139.

38. Backe MB, Moen IW, Ellervik $C$, et al. Iron regulation of pancreatic beta-cell functions and oxidative stress. Ann Rev Nutr. 2016;36:241-273. doi: https://doi.org/10.1146/annurev-nutr-071715-050939

39. Dongiovanni P, Ruscica M, Rametta R, et al. Dietary iron overload induces visceral adipose tissue insulin resistance. Am J Pathol. 2013;182(6):2254-2263. doi: https://doi.org/10.1016/j.ajpath.2013.02.019

40. Cejvanovic V, Kjær LK, Bergholdt HKM, et al. Iron induced rna-oxidation in the general population and in mouse tissue. Free Radic Biol Med. 2018;115:127-135. doi: https://doi.org/10.1016/j.freeradbiomed.2017.11.013

41. Tiongco RE, Arceo E, Clemente B, Pineda-Cortel MR. Association of maternal iron deficiency anemia with the risk of gestational diabetes mellitus: a metaanalysis. Arch Gynecol Obstet. 2019;299(1):89-95. doi: https://doi.org/10.1007/s00404-018-4932-0

42. Hershenfeld S, Ye C, Hanley AJ, et al. Serum Ferritin and Glucose Homeostasis in Women with Recent Gestational Diabetes. Can J Diabetes. 2019;43:567-572 doi: https://doi.org/10.1016/j.jcjd.2019.06.003

43. WHO recommendations on antenatal care for a positive pregnancy experience. World Health Organization. (2016). Available from: www.who.int/reproductivehealth/publications/maternal_ perinatal_health/anc-positive-pregnancy-experience/en/

44. Zhu B, Liang C, Xia X, et al. Iron-Related Factors in Early Pregnancy and Subsequent Risk of Gestational Diabetes Mellitus: the Ma'anshan Birth Cohort (MABC) Study. Biol Trace Elem Res. 2019;191(1):45-53. doi: https://doi.org/10.1007/s12011-018-1595-4

45. Liu X, Pang J. A retrospective study of supplemental iron intake in singleton pregnancy women with risk of developing gestational diabetes mellitus. Medicine. 2018;97(26):e10819. doi: https://doi.org/10.1097/MD.0000000000010819

\section{ИНФОРМАЦИЯ ОБ АВТОРАХ [AUTHORS INFO]}

*Торосян Айарпи Овсеповна, аспирант [Ayarpi O. Torosyan, PhD student]; адрес: Россия, 117198, г. Москва, ул. Миклухо-Маклая, д. 6 [address: 6, Miklukho-Maklaya street, 117198 Moscow, Russia]; ORCID: https://orcid.org/0000-0003-3403-1895; eLibrary SPIN: 5278-5585; e-mail: dr.arev@gmail.com

Логинова Екатерина Владимировна, аспирант [Ekaterina V. Loginova, PhD student];

ORCID: https://orcid.org/0000-0003-0274-1729; SPIN: 2818-8941; e-mail: katya.loginova@mail.ru

Гагаев Челеби Гасанович, д.м.н., профессор [Chelebi G. Gagaev, MD, PhD, Professor]; ORCID: https://orcid.org/0000-0001-7213-5981; eLibrary SPIN: 7414-7080; e-mail: gasanovich@gmail.com

\section{ЦИТИРОВАТЬ:}

Торосян А.О., Логинова Е.В., Гагаев Ч.Г. Роль показателей обмена железа в прогнозировании гестационного сахарного диабета // Сахарный диабет. — 2021. — Т. 24. — №4. — C. 365-370. doi: https://doi.org/10.14341/DM12502

\section{TO CITE THIS ARTICLE:}

Torosyan AO, Loginova EV, Gagaev ChG. The role of iron metabolism indicators in predicting gestational diabetes mellitus. Diabetes Mellitus. 2021;24(4):365-370. doi: https://doi.org/10.14341/DM12502 